\title{
El impacto de los desastres naturales y el cambio climático en el desplazamiento forzado de personas
}

\section{The Impact of Natural Disasters and Climate Change in the Forced Displacement of Persons}

Isabel Victoria Cabrera Rivera*

DOI: http://dx.doi.org/10.21503/lex.v16i21.1557

* Abogada y magíster en Derechos Humanos y Derecho Internacional Humanitario por el American University Washington College of Law (USA), maestría ejecutiva en Administración de Negocios por la Universidad Alas Peruanas (Perú), egresada del Doctorado en Educación de la Universidad Enrique Guzmán y Valle (Perú), egresada del Doctorado en Medio Ambiente y Desarrollo Sostenible de la Universidad Inca Garcilaso de la Vega (Perú), Posdoctorado en Ciencias de la Educación de la Universidad Enrique Guzmán y Valle (Perú). Docente de la Universidad Multiversidad Mundo Real Edgar Morín (México).

Correo electrónico: victoria_c24@hotmail.com

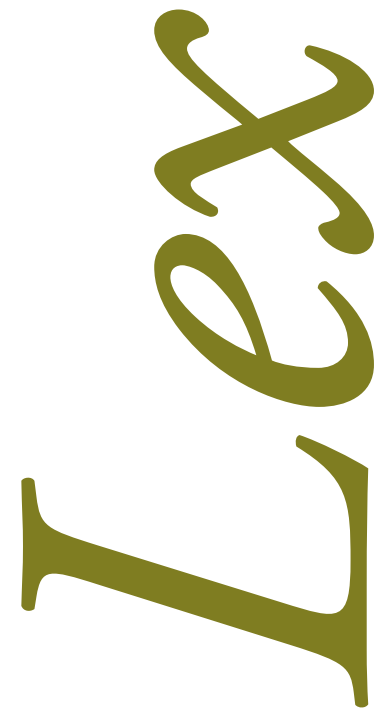




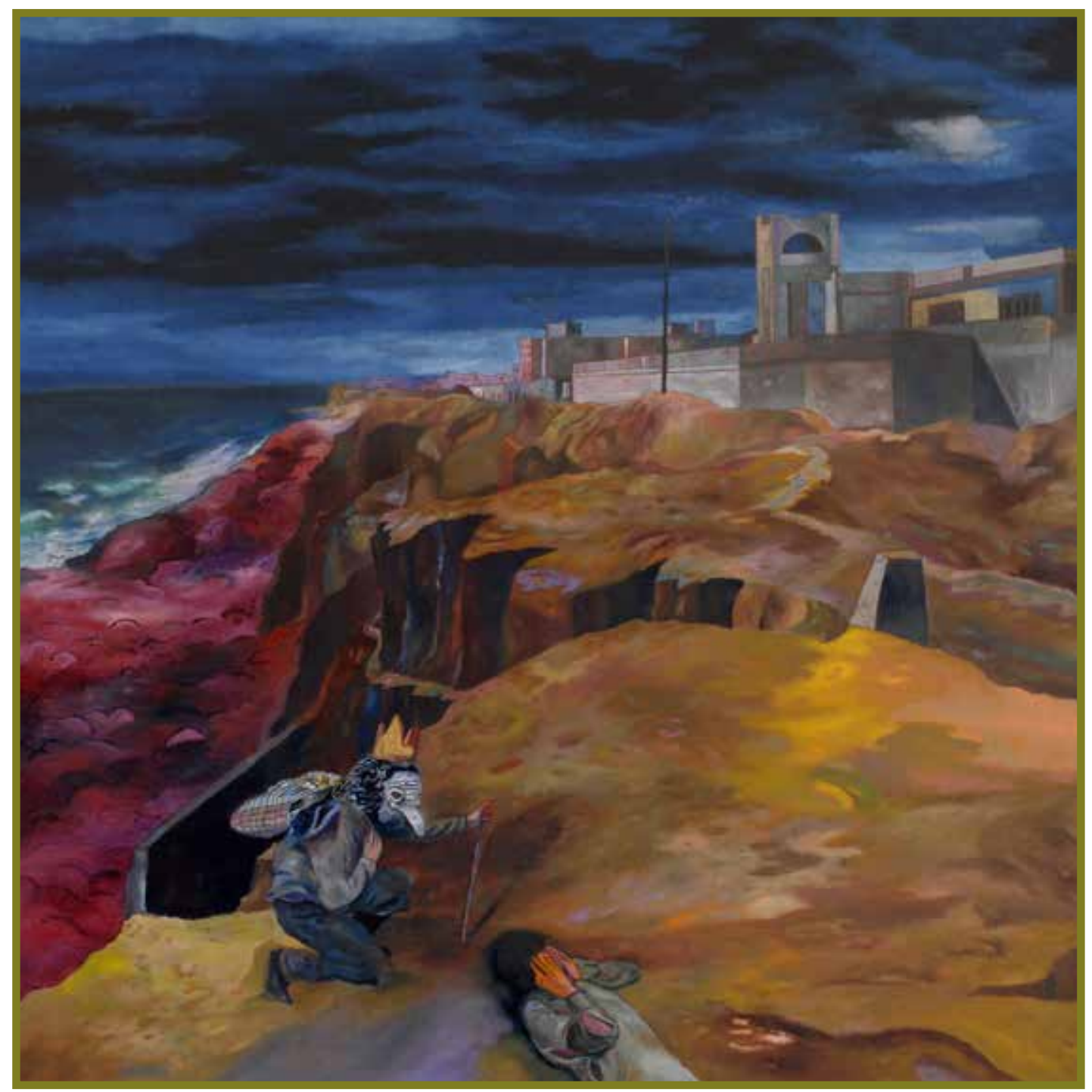

Los extramuros del mundo. Enrique Polanco.

https://www.facebook.com/enriquepolancopintor/ 


\section{RESUMEN}

En las últimas tres décadas, el cambio climático ha sido un tema que ha estado en constante discusión; sin embargo, hasta los últimos años se ha introducido en el debate lo concerniente a los desplazados ambientales. En este breve ensayo se abordará el impacto de los desastres naturales provocados por el cambio climático en el desplazamiento de personas, así como el marco jurídico que regula la figura de los refugiados, para de esta forma ver si se puede extender dicha protección, así como los impedimentos para ello.

Palabras clave: el impacto de los desastres naturales, cambio climático, desplazamiento forzado.

\section{ABSTRACT}

In the last three decades, the climate change has been an issue that has been in constant discussion, however, until recent years has been introduced in the debate concerning the displaced environmental. This brief essay will address the impact of natural disasters caused by climate change on the movement of people, as well as the legal framework that regulates the figure of refugees, in order to see if this protection can be extended, as well as the impediments to it.

Key words: the impact of natural disasters, climate change, forced displacement. 


\section{INTRODUCCIÓN}

Con la maximización de la producción en el mundo, las facilidades para tener vehículos, el uso de combustibles fósiles, la poca responsabilidad de las empresas en el tratamiento de sus residuos, así como el simple hecho de que ya somos más de siete mil millones de habitantes en el planeta, se ha provocado una descompensación en el clima de nuestro planeta, lo cual he tenido como consecuencia los cada vez más constantes desastres naturales a los cuales nos enfrentamos.

Lo anterior ha traído consigo problemas como el desplazamiento forzado de poblaciones que son afectadas en su calidad de vida por sequías, perdida de su hogar derivada de huracanes, terremotos, etc. Todo ello ha planteado desafíos a los Estados tanto económicos como jurídicos en el tratamiento que deben recibir las personas que migran por razones ambientales. A continuación, abordaremos las posibles obligaciones de los Estados, el estatuto que deben tener las personas víctimas de desplazamiento forzado por razones ambientales, así como los desafíos y propuestas que nacen de ese breve análisis.

Para ello, primero abordaremos el marco jurídico aplicable, después unas aproximaciones conceptuales, para luego abordar lo referente a los desplazados ambientales.

\section{MARCO JURÍDICO}

A nivel internacional y regional, hay un marco jurídico integrado por diversos instrumentos internacionales que protegen directa e indirectamente a los migrantes. Desde los que se enfocan en la protección de los derechos humanos de las personas con independencia de su nacionalidad, hasta aquellos que enfocan su protección en reducir los casos de apátridas, otorgar el estatuto de refugiado, por mencionar algunos.

En ese orden de ideas, enunciaremos brevemente los instrumentos internacionales más relevantes en la materia que nos ayudarán a definir conceptos importantes para fines del ensayo y para ver las obligaciones estatales en la materia. Empezaremos por aquellos que pertenecen al Sistema Universal de Protección de Derechos Humanos, para después abordar los referentes al Sistema Interamericano de Protección de Derechos Humanos. 
Por último, se mencionarán brevemente aquellos instrumentos internacionales en materia de compromisos de los Estados sobre el cambio climático, así como eventos organizados por organizaciones internacionales en donde los Estados hayan discutido sobre el tema, adoptado posturas y asumiendo compromisos.

\section{Internacional}

a) Convención sobre el Estatuto de los Refugiados.

b) Protocolo sobre el Estatuto de los Refugiados.

c) Convención sobre el Estatuto de los Apátridas.

d) Convención para Reducir los Casos de Apátrida.

e) Protocolo contra el tráfico ilícito de migrantes por tierra, mar y aire, que complementa la Convención de las Naciones Unidas contra la Delincuencia Organizada Transnacional, "Protocolo contra el tráfico ilícito de migrantes".

f) Convención de Viena sobre Relaciones Consulares.

g) Declaración Universal de Derechos Humanos.

h) Pacto Internacional de los Derechos Civiles y Políticos.

i) Pacto Internacional de los Derechos Económicos, Sociales y Culturales.

j) Convención Internacional sobre la Protección de los Derechos de Todos los Trabajadores Migratorios y de sus Familiares.

k) Los órganos encargados de supervisar el cumplimiento de los tratados en materia de derechos humanos son los comités convencionales, los cuales tienen naturaleza jurisdiccional.

1) Existen órganos más bien de carácter político, tales como el Consejo de Derechos Humanos.

m) Asimismo, la máxima autoridad en materia de migración, específicamente de refugiados, fue creada en 1950, y se trata del Alto Comisionado de las Naciones Unidas para los Refugiados (ACNUR).

\section{Regional}

a) Declaración Americana de los Derechos y Deberes del Hombre.

b) Convención Americana sobre Derechos Humanos.

c) Protocolo Adicional a la Convención Americana sobre Derechos Humanos en Materia de Derechos Económicos, Sociales y Culturales, "Protocolo de San Salvador". 
d) Convención Interamericana para Prevenir, Sancionar y Erradicar la Violencia contra la Mujer, "Convención de Belém do Pará".

e) Convención Interamericana sobre la Protección de los Derechos Humanos de las Personas Mayores.

f) Los dos máximos órganos del sistema son la Comisión Interamericana de Derechos Humanos (CIDH), órgano promotor y cuasi jurisdiccional; y la Corte Interamericana de Derechos Humanos (Corte IDH), órgano jurisdiccional y consultivo.

Una vez analizado el marco jurídico internacional, podemos desprender de dichos instrumentos aproximaciones conceptuales de los principales términos utilizados para este ensayo de investigación, pero antes debemos enunciar brevemente los instrumentos y conferencias relacionados con el cambio climático.

\section{Marco sobre cambio climático}

a) Conferencia Científica de las Naciones Unidas (Cumbre para la Tierra).

b) Convención Marco de las Naciones Unidas sobre el Cambio Climático.

c) Protocolo a la Convención Marco de Naciones Unidas sobre Cambio Climático (Protocolo de Kioto).

d) Se han desarrollado desde 1995, 23 conferencias sobre el cambio climático.

e) Programa de las Naciones Unidas para el Medio Ambiente.

f) Organización Meteorológica Mundial.

g) Actualmente existe un Grupo Intergubernamental de Expertos sobre el Cambio Climático (IPCC) que fue creado por la Organización Meteorológica Mundial (OMM) y el Programa de las Naciones Unidas para el Medio Ambiente (PNUMA).

h) Acuerdos de Paris.

\section{APROXIMACIONES CONCEPTUALES}

Para hablar de desplazamiento forzado de personas por razones ambientales, primero tenemos que hacer algunas precisiones conceptuales contenidas en instrumentos internacionales que ya citamos, o propuestas por organizaciones internacionales.

Precisado lo anterior, a nivel internacional no existe un consenso sobre una definición de migrante, por lo cual se retomará la propuesta por el Informe sobre Desplazados de la CIDH. En este sentido, se entenderá por "migrante internacional" toda persona que se encuentre por 
fuera del Estado del cual es nacional; y "migrante interno" para referirse a toda persona que se encuentre dentro del territorio del cual es nacional, pero por fuera del lugar en el que nació o donde reside habitualmente. ${ }^{1}$

La Organización Internacional para las Migraciones establece que los migrantes por causas naturales son "Las personas o grupos de personas que por culpa de los cambios medioambientales ineludibles, súbitos o progresivos, que afectan de forma negativa sus vidas o sus condiciones de vida, se ven obligadas a dejar sus hogares habituales, o deciden hacerlo voluntariamente. El desplazamiento puede ser temporal o permanente, en el interior del país o en el extranjero". La ONU estima que para el año 2050, el número de migrantes por causas ambientales podría rondar los 200 millones de personas; que la inmensa mayoría de esas personas provendría de países subdesarrollados; que dichas causas de la movilización serían principalmente por aumento en la temperatura global; que precisamente dichos países subdesarrollados son los que a nivel mundial menos aportan al fenómeno del calentamiento global y que las consecuencias de no brindarles la protección necesaria a esta cantidad de personas, que como cuestión de realidad sería mayor que los "refugiados ordinarios", serían nefastas no solo para los migrantes por causas ambientales sino para el resto del mundo. ${ }^{2}$

Desde el punto de vista de los derechos humanos, dicha movilidad de personas tendría efectos negativos en el sistema de salud "dificultando el tratamiento y erradicación de enfermedades infecto-contagiosas y aumentando la morbimortalidad".

En ese mismo orden de ideas, el artículo 1 de la Convención sobre el Estatuto de los Refugiados de 1951, modificada por el Protocolo sobre el Estatuto de los Refugiados de 1967, utiliza el término "refugiado" para aquellas personas que debido a fundados temores de ser perseguidas por motivos de raza, religión, nacionalidad, pertenencia a determinado grupo social u opiniones políticas, se encuentran fuera del país de su nacionalidad; o que, careciendo de nacionalidad y hallándose, fuera del país donde tuviera su residencia habitual, no pueda o, a causa de dichos temores, no quiera regresar a él. ${ }^{3}$

En lo que refiere a los desplazados ambientales, el ACNUR ha referido que cuando hablamos de desplazamiento forzoso, generalmente lo asociamos a aquellas personas que han debido abandonar sus hogares por culpa de los conflictos armados o la persecución política, ideológica, religiosa, étnica, etc. Sin embargo, en los últimos ańos, una buena parte de las

\footnotetext{
Comisión Interamericana de Derechos Humanos, Movilidad humana: estándares interamericanos, 2015, http://www.oas. org/es/cidh/informes/pdfs/MovilidadHumana.pdf.

2 ONU, “ONU: En el 2050 podría haber 200 millones de desplazados por cambio climático", La Información, (2016), https:/www.lainformacion.com/medio-ambiente/preservacion/onu-en-el-2050-podria-haber-200-millones-de-desplazados-por-cambio-climatico_8tjuEWMCynQo255XtNLh31/

3 ACNUR, "Convención sobre el Estatuto de los Refugiados" 1951.
} 
personas que dejan su hogar lo hacen por razones medioambientales, es decir, relacionadas con las condiciones de los entornos y el desequilibrio planetario. ${ }^{4}$

El término "solicitante de asilo" hace relación a la persona que solicita el reconocimiento de la condición de refugiado y cuya solicitud todavía no ha sido evaluada en forma definitiva en el país de acogida. ${ }^{5}$

Por otro lado, hay otros conceptos que hay que definir y que no pertenecen al ámbito legal pero que para nuestro ensayo son imprescindibles. Entre ellos está el cambio climático, el cual se va a entender como la alteración del clima mundial que nosotros, los seres humanos, estamos ocasionando como consecuencia del consumo de combustibles fósiles, la tala de bosques y otras prácticas que incrementan la concentración de gases de efecto invernadero (GEI) en la atmósfera, es decir, el cambio climático se puede atribuir "directa o indirectamente a las actividades humanas que alteran la composición de la atmósfera global y que se suma a la variabilidad del clima natural observada durante períodos de tiempo comparables. ${ }^{6}$

Asimismo, se entiende por desastre natural como un evento provocado por la naturaleza de la suficiente magnitud, que altera la estructura básica y el funcionamiento normal de una sociedad o comunidad, ocasionando víctimas y daños o pérdidas de bienes materiales, infraestructura, servicios esenciales o medios de sustento a escala o dimensión más allá de la capacidad normal de las comunidades o instituciones afectadas para enfrentarlas sin ayuda. También se considera si existe una perturbación ecológica abrumadora que acaece a escala suficiente para que se necesite auxilio. Todos los desastres naturales son fenómenos que, en principio, no son producidos por la acción directa del hombre. Sin embargo, como veremos en el siguiente apartado, esta última afirmación es relativa. ${ }^{7}$

\section{EL CAMBIO CLIMÁTICO Y LOS DESASTRES NATURALES}

El cambio climático ha sido algo común en la historia de nuestro planeta. Los glaciares y diversas pruebas geológicas han demostrado las fluctuaciones en la temperatura del mundo, las cuales solían ser provocadas por el cambio de las corrientes de los océanos, las erupciones volcánicas, impactos de cuerpos celestes, la actividad solar u otros factores naturales. ${ }^{8}$

\footnotetext{
ACNUR, “Convención sobre el Estatuto de los Refugiados”...

Comisión Interamericana de Derechos Humanos, Movilidad humana: estándares interamericanos...

ONU, “Convención Marco de las Naciones Unidas sobre Cambio Climático”, 1992.

Lindsey Jones, "Cambio climático y riesgo de desastres", en Futuro marco para la reducción del riesgo de desastres, ed. por Jan Kellett y Tom Mitchell (Alianza Clima y Desarrollo (CDKN) e Instituto de Desarrollo Exterior (ODI), 2014), 41-80.

8 ACNUR, "Cambio climático, desastres naturales y desplazamiento humano: la perspectiva del ACNUR". Actualización 2008, http://www.unhcr.org/497891022.pdf
} 
Sin embargo, en los últimos años el cambio climático ha tenido como causas no solo los fenómenos que periódicamente se daban en nuestro planeta, sino que se ha demostrado la estrecha relación entre la alteración del clima y la actividad humana.

Lo anterior ha tenido como consecuencia que los desastres naturales sean más comunes, tales como ciclones o huracanes tropicales, inundaciones, sequías, tormentas de polvo, terremotos, tsunamis, erupciones volcánicas, avalanchas de nieve, glaciales, hundimientos, contaminación del agua, quema de carbón, erosión costera, malas cosechas, plagas de insectos, declives de arrecifes de coral, lluvia ácida, contaminación, efecto invernadero, aumento del nivel del mar, fenómeno del nińo, descenso de la capa de ozono, impactos de asteroides y auroras boreales, por mencionar algunos. ${ }^{?}$

Todos ellos, en mayor o menor medida, han aumentado su frecuencia en los últimos años, ya que la contaminación que generamos es cada vez más nociva para nuestro planeta e impacta en mayor medida a las zonas del planeta que son más vulnerables por condiciones de pobreza o su ubicación geográfica.

El aumento de dichos fenómenos trae consigo variados problemas, uno de los cuales es el desplazamiento forzado de las personas afectadas por los desastres naturales. Ello plantea muchas y variadas interrogantes, pues al ser personas desplazadas por causas de fuerza mayor, podrían ser acreedoras a una especial protección internacional. Para dilucidar ello, primero se abordarán algunos casos recientes de desplazamiento forzado causado por el cambio climático y los desastres naturales que sustentan la pertinencia de una categoría como refugiado ambiental.

\section{CASOS DE DESPLAZAMIENTOS POR DESASTRES NATURALES}

Un estudio de la Oficina de Coordinación de Asuntos Humanitarios de las Naciones Unidas (OCHA, por sus siglas en inglés) y el Centro de Vigilancia de los Desplazamientos Internos del Consejo Noruego para Refugiados señala que, todos los ańos, los desastres relacionados con los fenómenos climáticos ya desplazan a millones de personas. ${ }^{10}$

Es así que en las últimas dos décadas, el número de desastres naturales registrados se ha duplicado de 200 a más de 400 por ańo, los cuales son, en su mayoría, desastres relacionados con el clima.

9 Literatura Cubana, “Desastres naturales”, 2009, 101-14, http://www.sld.cu/galerias/pdf/sitios/urgencia/10desastres_naturales.pdf

10 Vikram Kolmannskog and Lisetta Trebbi, "Cambio climático, desastres naturales y desplazamiento: un enfoque múltiple para resolver las brechas de protección”, International Review of the Red Croos, n. ${ }^{\circ} 879$ (2010). 
En la región, por ejemplo, uno de los últimos desastres naturales que generaron un gran número de desplazados fue el terremoto de Haití en 2010. Según la ONU, tan solo en enero de 2010 causó la muerte de más de 200000 personas y dejó sin hogar al menos a 1,5 millones más. Además, 60000 personas fueron desplazadas a causa de ello. ${ }^{11}$

Apenas en 2017, en República Dominicana, 14000 personas fueron desplazadas a causa del devastador huracán María. Asimismo, países de regiones económicamente más avanzadas también sufren ya de desplazamiento por estas causas, lo cual demuestra que no es exclusivo de países con índice de desarrollo humano bajo. El ejemplo es Italia, que en 2016 fue sacudido por un terremoto que en la región de Pescara dejó 7500 desplazados. ${ }^{12}$

Perú tampoco está exento de desplazados ambientales, tan solo en 2017 hubo 20000 desplazados en la ciudad de Piura a causa de las lluvias provocadas por el fenómeno El Niño, que año tras año es más fuerte y devastador para nuestro país. ${ }^{13}$ Si bien estos últimos son en su mayoría desplazados internos, igual su condición exige un marco jurídico protector interno que facilite su retorno o la ayuda para su establecimiento.

Los datos mencionados son de fenómenos naturales relativamente recientes, pero hay estudios que demuestran que hasta el año de 2008 los desastres naturales causaron el desplazamiento de 36 millones de personas, de las cuales 20 millones fueron por desastres naturales de aparición repentina relacionados con el clima. Además de ello, hay conflictos armados que provocan desplazados y cuyo origen está relacionado con guerras por fuentes de energía, agua, etc., recursos que escasean a causa del cambio climático.

Por último, como dato más reciente, el Banco Mundial estima que para 2050 podría haber más de 140 millones de migrantes internos por motivos climáticos, y agrega que estas personas se verán desplazadas por las sequías, las malas cosechas, el aumento del nivel del mar y las mareas de tormenta. ${ }^{14}$ Ante ese escenario, en el caso de los migrantes internacionales e internos, ¿qué responsabilidad y obligaciones tienen los Estados? Eso se tratará de responder en el apartado siguiente.

\section{OBLIGACIONES DE LOS ESTADOS RESPECTO A LOS DESPLAZADOS AMBIENTALES}

Para hablar de obligaciones de los Estados sobre los desplazados ambientales, se tendría que reconocer a los migrantes internacionales el estatus de refugiado, el cual hace referencia a

11 Noticias ONU, “Unos 60000 desplazados por el terremoto de 2010 en Haití continúan en campamentos”, 2016, https://news.un.org/es/story/2016/01/1348731.

12 Lucía Magi, "7 500 italianos siguen desplazados un año después del terremoto de Amatrice”, El País, 2017.

13 JCG, "Gobierno peruano estima 20 mil desplazados por lluvias en Piura," Made for Minds (2017).

14 Banco Mundial, "El rostro humano de las migraciones provocadas por impactos climáticos”, 2018. 
un estatus legal que se posee por encontrarse en un supuesto de hecho que diversos tratados internacionales, firmados por los Estados, reconocen. Este estatus, según ACNUR, se obtiene por circunstancias objetivas relacionadas con situaciones en las que las personas se ven obligadas a abandonar su hogar, la mayoría de las veces por conflictos armados o situaciones generalizadas de violencia, y por persecución dentro de sus Estados de origen y el temor fundado de regresar, por lo cual, en principio, el término está ceñido a personas que se desplazan por guerras, violencia o persecución. ${ }^{15}$

Es así que, hasta hoy, el término de refugiado ambiental no existe en el derecho internacional, por lo cual los Estados no tienen la obligación de reconocer dicho estatus a quienes abandonan sus lugares de origen por causas directamente atribuibles a cuestiones ambientales, dejándolos en estado de indefensión.

Hay muchas y variadas razones para poder construir un marco jurídico internacional que permita reconocer el estatus de refugiado a los desplazados ambientales, entre ellas la responsabilidad directa de los Estados industrializados en el cambio climático en el mundo, lo cual se vuelve una presunción de que la causa del desplazamiento es la irresponsabilidad de los Estados y de las grandes empresas internacionales al contribuir al cambio climático. Por otro lado, uno de los principios del derecho internacional que es la cooperación, también se esboza como una razón más para crear el marco jurídico protector.

Si los fenómenos naturales cada vez más agresivos a causa del cambio climático causado por los humanos provocan desplazados, lo lógico es que dichos Estados asuman obligaciones internacionales sobre ello. Hasta ahora, solo algunos países de manera voluntaria reciben refugiados por causas ambientales.

La Organización Internacional para las Migraciones ha reconocido tres tipos de migrantes ambientales:

- Inmigrantes por emergencia ambiental: las personas que huyen temporalmente debido a un desastre ambiental o a eventos ambientales bruscos.

- Inmigrantes forzosos ambientales, migrantes forzados: las personas que tienen que abandonar sus hogares debido al deterioro de las condiciones ambientales.

- Inmigrantes motivados por el ambiente también conocidos como migrantes económicos inducidos por el ambiente: las personas que optan por dejar sus hogares para evitar posibles problemas futuros.

15 Los Estados Parte, "Protocolo sobre el Estatuto de los Refugiados", 1186, n. ${ }^{\circ}$ XLI (1967). 
Dentro de esa categorización nosotros sugerimos que cualquier persona que entre en cualquiera de los supuestos tenga derecho a que le sea reconocido el estatus de refugiado con todos los derechos y beneficios que tiene en el derecho internacional. Para ello, se tendría que emprender una modificación al marco jurídico internacional que ya existe, en este caso, a la Convención sobre el Estatuto de los Refugiados y al Protocolo sobre el Estatuto de los Refugiados. Todo ello con fundamento en los instrumentos internacionales en materia de derechos humanos, que mencionan, entre otras cosas, que los derechos humanos se reconocen con independencia del país de origen, por lo cual, en principio, cualquier Estado estaría obligado a facilitar las condiciones para su establecimiento.

Asimismo, otra opción sería un nuevo instrumento internacional con obligaciones para los Estados y derechos para los refugiados ambientales, que atienda las necesidades específicas que tiene un desplazado por razones ambientales. Esto último parece un tanto difícil. Hace apenas unos meses, el presidente de los Estados Unidos de América detuvo las negociaciones para estar en los Acuerdos de Paris, lo cual representó un retroceso para la materia.

Por otro lado, en Latinoamérica la protección de los migrantes es cada vez más amplia, y la CIDH y Corte IDH podrían en futuros casos obligar con sus decisiones a los Estados a extender esa protección a las personas que se desplazan por no tener las condiciones ambientales necesarias para garantizar una vida digna.

Ahora bien, en lo que toca a los desplazados internos, los Estados en sus jurisdicciones internas han tomado medidas para ayudar a estas personas a establecerse en nuevos sitios o a poder retornar a sus lugares de origen. Un ejemplo de ello son México y Perú, que cada que se dan huracanes y el fenómeno El Niño implementan programas de reconstrucción de la vida para los afectados. Los problemas parecen ir más allá de las fronteras de los Estados, pues hay países que se perfilan como inhabitables en las próximas décadas, ya sea por el aumento del nivel de mar causado por el calentamiento global, o por su posición geográfica cada vez más vulnerable a los desastres naturales.

\section{CONCLUSIONES}

Podemos concluir que existe un marco jurídico que podría proteger a los desplazados ambientales; sin embargo, se necesita hacer adecuaciones ya que el derecho internacional, en principio, no los protege, por lo que tenemos situaciones legales y sociopolíticas donde a simple vista el refugiado ambiental resulta inviable.

Por otro lado, estamos frente a una realidad innegable: la de millones de desplazados por causas ambientales que no gozan de una protección internacional que les garantice el ejercicio de sus derechos humanos. Los impedimentos para alcanzar ese marco jurídico ideal son varios, entre ellos, un problema de operatividad, pues otorgar el estatuto de refugiados debe 
obedecer a ciertos criterios objetivos que son difíciles de acreditar en el caso de los desplazados ambientales.

Los retos son aún más, no solo radican en otorgar una protección jurídica amplia a quienes lo necesitan, sino eliminar de raíz el problema que es el cambio climático, para lo cual se necesita la cooperación de las empresas, las organizaciones y, sobre todo, de los Estados, en especial aquellos que por sus niveles de industrialización contribuyen en mayor medida a el cambio climático.

\section{REFERENCIAS}

- ACNUR. "Cambio climático, desastres naturales y desplazamiento humano: la perspectiva del ACNUR”. Actualización 2008, 1-14. http://www.unhcr.org/497891022.pdf.

“Convención sobre el Estatuto de los Refugiados,” 1951.

- Banco Mundial. "El rostro humano de las migraciones provocadas por impactos climáticos”. Banco Mundial, 2018.

- Comisión Interamericana de Derechos Humanos. Movilidad humana: estándares interamericanos, 2015. http://www.oas.org/es/cidh/informes/pdfs/MovilidadHumana.pdf

- JCG. "Gobierno peruano estima 20 mil desplazados por lluvias en Piura". Made for Minds. 2017.

- Jones, Lindsey. "Cambio climático y riesgo de desastres". En Futuro marco para la reducción del riesgo de desastres, ed. por Jan Kellett y Tom Mitchell, 41-80. Alianza Clima y Desarrollo (CDKN) e Instituto de Desarrollo Exterior (ODI), 2014.

- Kolmannskog, Vikram and Lisetta Trebbi. "Cambio climático, desastres naturales y desplazamiento: un enfoque múltiple para resolver las brechas de protección”. International Review of the Red Croos, n. 879 (2010): 307-328.

- Literatura Cubana. "Desastres naturales" 2009. http://www.sld.cu/galerias/pdf/sitios/urgencia/10desastres_naturales.pdf

- Los Estados Parte. "Protocolo sobre el Estatuto de los Refugiados" 1186, n. ${ }^{\circ}$ XLI (1967): $1-4$.

- Magi, Lucía. "7 500 italianos siguen desplazados un año después del terremoto de Amatrice". El Pais, 2017. 
- ONU. "Convención Marco de las Naciones Unidas sobre Cambio Climático". 1992.

“ONU: En el 2050 podría haber 200 millones de desplazados por cambio climático". La Información (2016). https://www.lainformacion.com/medio-ambiente/ preservacion/onu-en-el-2050-podria-haber-200-millones-de-desplazados-por-cambio-climatico_8tjuEWMCynQo255XtNLh31/

- ONU, Noticias. "Unos 60000 desplazados por el terremoto de 2010 en Haití continúan en campamentos". 2016. https://news.un.org/es/story/2016/01/1348731.

Recibido: 18/03/18

Aprobado: 27/04/18 